\title{
RECOVERING SINGULAR DIFFERENTIAL OPERATORS ON NONCOMPACT STAR-TYPE GRAPHS FROM WEYL FUNCTIONS
}

\author{
V. YURKO
}

\begin{abstract}
Bessel-type differential operators on noncompact star-type graphs are studied. We establish properties of the spectral characteristics and then we investigate the inverse problem of recovering the operator from the so-called Weyl vector. For this inverse problem we prove a uniqueness theorem and propose a procedure for constructing the solution using the method of spectral mappings.
\end{abstract}

\section{Introduction}

Analysis on graphs and other similar structures has been developing for quite some time due to various applications in applied sciences. In particular in recent years it has experienced a significant boost in terms of new applications arising and new methods developed and studied.

In this paper we present the solution of an inverse spectral problem for Bessel-type differential operators on noncompact star-type graphs. This inverse problem consists in recovering the potential of the Bessel operator on a graph from the given spectral characteristics. We recall that differential operators on graphs (networks, trees) often appear in mathematics, mechanics, physics, geophysics, physical chemistry, biology, electronics, nanoscale technology and other branches of natural sciences and engineering (see [1]-[4] and the references therein). Recently there has been increasing interest in spectral theory of differential equations on graphs (for a good review of such publications see [5]-[6]). Most of the works in this direction are devoted to the so-called direct problems of studying properties of the spectrum and the root functions. Inverse spectral problems, because of their nonlinearity, are more difficult for investigating. For Sturm-Liouville operators on compact graphs inverse problems were studied in [7]-[14] and other works. Noncompact case for Sturm-Liouville operators was considered in [15]-[17]. Bessel operators on graphs have not been studied yet.

2000 Mathematics Subject Classification. 34A55 34B45 34B40 47E05.

Key words and phrases. Singular differential equations, noncompact graphs, inverse spectral problems, method of spectral mappings. 
In this paper we provide a formulation and the solution of the inverse problem of recovering singular potential of the Bessel operator on noncompact star-type graphs which is a natural generalization of the well-known inverse problems for differential operators on an interval (see the monographs [18]-[27] and the references therein). As the main spectral characteristic we introduce and study the so-called Weyl vector which is a generalization of the Weyl function (m-function) for the classical Sturm-Liouville operator (see [28]). We show that the specification of the Weyl vector uniquely determines the potential, and we provide a constructive procedure for the solution of the inverse problem from the given Weyl vector. Here we face with a singular case having more complicated behavior of the spectrum which leads to new qualitative difficulties for studying direct and inverse problems. For definiteness, we confine ourselves to graphs with one infinite edge.

For studying the inverse problem on noncompact graphs we develop the ideas of the method of spectral mappings [23], [25]. This method allows one to solve inverse problems for a wide class of operators on graphs. Note that the obtained results are valid not only for the selfadjoint case but also for the non-selfadjoint one when the potential is a complex-valued function on the graph.

The paper is organized as follows: In section 2 we introduce the main notions and formulate a boundary value problem. In order to define boundary conditions at singular boundary vertices we use ideas from [29]. In Section 3 properties of the spectrum are studied. In particular, Theorems 1-5 describe the continuous and the discrete spectrum and connections between them. In Section 4 the solution of the inverse problem is given.

\section{Boundary value problem}

Consider a noncompact star-type graph $T$ in $\mathbf{R}^{N}$ with the set of vertices $V=\left\{v_{0}, \ldots, v_{p}\right\}$, and the set of edges $\mathscr{E}=\left\{e_{0}, \ldots, e_{p}\right\}$, where $e_{j}=\left[v_{j}, v_{0}\right], j=\overline{1, p}$, are finite segments, and $e_{0}=$ $\left[v_{0}, v_{p+1}\right)$ is an infinite ray, $v_{p+1}:=\infty$.

Let $l_{j}$ be the length of the edge $e_{j}, j=\overline{1, p}$. Each edge $e_{j}, j=\overline{1, p}$, is parameterized by the parameter $x_{j} \in\left[0, l_{j}\right]$ such that the initial point $v_{j}$ corresponds to $x_{j}=0$, and the terminal point $v_{0}$ corresponds to $x_{j}=l_{j}$. The ray $e_{0}=\left[v_{0}, \infty\right)$ is parameterized by the parameter $x_{0} \epsilon$ $[0, \infty)$ such that $x_{0}=0$ corresponds to the vertex $v_{0}$.

A function $Y$ on $T$ may be represented as $Y=\left\{y_{j}\right\}_{j=\overline{0, p}}$, where the function $y_{j}\left(x_{j}\right)$, is defined on the edge $e_{j}$. Consider the differential equation on $T$ :

$$
\ell_{j} y_{j}\left(x_{j}\right):=-y_{j}^{\prime \prime}\left(x_{j}\right)+Q_{j}\left(x_{j}\right) y_{j}\left(x_{j}\right)=\lambda y_{j}\left(x_{j}\right), \quad Q_{j}\left(x_{j}\right)=\frac{\omega_{j}}{x_{j}^{2}}+q_{j}\left(x_{j}\right), \quad j=\overline{0, p},
$$

where $\lambda$ is the spectral parameter, $\omega_{j}$ are real numbers, and $Q=\left\{Q_{j}\right\}_{j=\overline{0, p}}$ is a real-valued function on the graph $T$. For definiteness, we assume that $\omega_{j}=v_{j}^{2}-1 / 4, v_{j}>0, v_{j} \notin \mathbf{N}, v_{0}=$ 
$1 / 2, q_{j}\left(x_{j}\right) x_{j}^{1-2 v_{j}}$ are integrable on $e_{j}$ (other cases are treated similarly). The function $Q$ on the graph $T$ is called the potential.

In order to define boundary conditions at the boundary vertices $v_{j}, j=\overline{1, p}$, we will use ideas from [29]. For this purpose, we consider the Bessel-type fundamental system of solutions $\left\{S_{j m}\left(x_{j}, \lambda\right)\right\}_{m=1,2}$ of equation (1) on the edge $e_{j}, j=\overline{1, p}$, with the following properties (see [30]):

(a) For each fixed $x_{j} \in\left(0, l_{j}\right)$, the functions $S_{j m}^{(\xi)}\left(x_{j}, \lambda\right), \xi=0,1$, are entire in $\lambda$.

(b) For $x_{j} \rightarrow 0$,

$$
S_{j m}\left(x_{j}, \lambda\right) \sim c_{j m} x_{j}^{\mu_{j m}},
$$

where $\mu_{j m}=(-1)^{m} v_{j}+1 / 2, c_{j 1} c_{j 2}=\left(2 v_{j}\right)^{-1}$.

(c) The following relation holds

$$
\left\langle S_{j 1}\left(x_{j}, \lambda\right), S_{j 2}\left(x_{j}, \lambda\right)\right\rangle \equiv 1,
$$

where $\langle y(x), \tilde{y}(x)\rangle:=y(x) \tilde{y}^{\prime}(x)-y^{\prime}(x) \tilde{y}(x)$ is the Wronskian of $y$ and $\tilde{y}$.

Similar to [29] we introduce the linear forms

$$
\sigma_{j k}\left(y_{j}\right):=(-1)^{k-1}\left\langle y_{j}\left(x_{j}\right), S_{j, 3-k}\left(x_{j}, \lambda\right)\right\rangle_{\mid x_{j}=0}, \quad k=1,2, j=\overline{1, p} .
$$

It follows from (2) that

$$
\sigma_{j k}\left(S_{j m}\right)=\delta_{k m}, \quad m, k=1,2,
$$

where $\delta_{k m}$ is the Kronecker symbol. We note that for the classical Sturm-Liouville equation on $e_{j}$ one has $v_{j}=1 / 2$ (i.e. $\omega_{j}=0$ ); hence in this case $\sigma_{j k}\left(y_{j}\right)=y_{j}^{(k-1)}(0), k=1,2$, i.e., the boundary functionals have the classical form. Let $h=\left[h_{j}\right]_{j=\overline{1, p}}$ be the vector, where $h_{j}$ are real numbers. Denote $U_{j}\left(y_{j}\right)=\sigma_{j 2}\left(y_{j}\right)-h_{j} \sigma_{j 1}\left(y_{j}\right), V_{j}\left(y_{j}\right)=\sigma_{j 1}\left(y_{j}\right), \varphi_{j 1}\left(x_{j}, \lambda\right)=S_{j 1}\left(x_{j}, \lambda\right)+$ $h_{j} S_{j 2}\left(x_{j}, \lambda\right), \varphi_{j 2}\left(x_{j}, \lambda\right)=S_{j 2}\left(x_{j}, \lambda\right)$. In view of (3), $\sigma_{j 1}\left(\varphi_{j 1}\right)=1, \sigma_{j 2}\left(\varphi_{j 1}\right)=h_{j}, U_{j}\left(\varphi_{j 1}\right)=0$. It follows from (2) that

$$
\left\langle\varphi_{j 1}\left(x_{j}, \lambda\right), \varphi_{j 2}\left(x_{j}, \lambda\right)\right\rangle \equiv 1
$$

Consider equation (1) on $T$, where

$$
y_{j}, y_{j}^{\prime} \in A C\left(0, l_{j}\right], j=\overline{1, p} ; \quad y_{0}, y_{0}^{\prime} \in A C_{l o c}[0, \infty),
$$

and $Y=\left\{y_{j}\right\}_{j=\overline{0, p}}$ satisfy the following matching conditions in the internal vertex $v_{0}$ :

$$
\left.\begin{array}{r}
y_{j}\left(l_{j}\right)=y_{0}(0) \quad \text { for all } \quad j=\overline{1, p} \quad \text { (continuity condition) }, \\
\sum_{j=1}^{p} y_{j}^{\prime}\left(l_{j}\right)=y_{0}^{\prime}(0) \quad \text { (Kirchhoff's condition). }
\end{array}\right\}
$$


The matching conditions (5) are called the standard matching conditions. Moreover, we additionally require that the function $Y=\left\{y_{j}\right\}_{j=0, p}$ satisfies the following boundary conditions at the boundary vertices:

$$
U_{j}\left(y_{j}\right)=0, \quad j=\overline{1, p}
$$

We consider the operator

$$
L^{\prime}: \quad D\left(L^{\prime}\right) \rightarrow \mathscr{L}_{2}(T), \quad Y=\left\{y_{j}\right\}_{j=\overline{0, p}} \rightarrow L^{\prime} Y:=\left\{\ell_{j} y_{j}\right\}_{j=\overline{0, p}}
$$

where the domain of definition $D\left(L^{\prime}\right)$ consists of functions $Y=\left\{y_{j}\right\}_{j=\overline{0, p}}$ satisfying (4)-(6), and $y_{0}, \ell_{0} y_{0} \in \mathscr{L}_{2}(0, \infty), \ell_{j} y_{j} \in \mathscr{L}_{2}\left(0, l_{j}\right), j=\overline{1, p}$. We denote the corresponding boundary value problem (1), (4)-(6) by $L$.

\section{Properties of the spectrum}

Let $\lambda=\rho^{2}$, and let for definiteness $\operatorname{Im} \rho \geq 0$. Put $\Omega_{0}=\{\rho: \operatorname{Im} \rho>0\}, \Omega=\{\rho: \operatorname{Im} \rho \geq$ $0, \rho \neq 0\}$. Denote by $\Pi$ the $\lambda$-plane with the cut $\lambda \geq 0$, and $\Pi_{1}=\bar{\Pi} \backslash\{0\}$; notice that here $\Pi$ and $\Pi_{1}$ must be considered as subsets of the Riemann surface of the square-root-function. Then, under the map $\rho \rightarrow \rho^{2}=\lambda, \Pi_{1}$ corresponds to the domain $\Omega$, and $\Pi$ corresponds to $\Omega_{0}$. Denote by $e\left(x_{0}, \rho\right), x_{0} \geq 0$, the Jost solution of equation (1) on the edge $e_{0}$ (see [23, Sec. 2.1]).

Lemma 1. The function $e\left(x_{0}, \rho\right)$ has the following properties:

(1) For each fixed $x_{0} \geq 0$, and $v=0,1$, the functions $e^{(v)}\left(x_{0}, \rho\right)$ are analytic for $\rho \in \Omega_{0}$, and are continuous for $\rho \in \Omega$.

(2) For $x_{0} \rightarrow \infty, v=0,1$,

$$
e^{(v)}\left(x_{0}, \rho\right)=(i \rho)^{v} \exp \left(i \rho x_{0}\right)(1+o(1)) .
$$

For $\rho \in \Omega_{0}, e\left(x_{0}, \rho\right) \in \mathscr{L}_{2}(0, \infty)$. Moreover, $e\left(x_{0}, \rho\right)$ is the unique solution of (1) on $e_{0}$ (up to a multiplicative constant) having this property.

(3) $\operatorname{For}|\rho| \rightarrow \infty, \rho \in \Omega, v=0,1$,

$$
e^{(v)}\left(x_{0}, \rho\right)=(i \rho)^{v} \exp \left(i \rho x_{0}\right)\left(1+O\left(\rho^{-1}\right)\right)
$$

uniformly for $x_{0} \geq 0$.

(4) For real $\rho \neq 0$, the functions $e\left(x_{0}, \rho\right)$ and $e\left(x_{0},-\rho\right)$ form a fundamental system of solutions for equation (1) on the edge $e_{0}$, and

$$
\left\langle e\left(x_{0}, \rho\right), e\left(x_{0},-\rho\right)\right\rangle=-2 i \rho .
$$


(5) For real $\rho \neq 0, \overline{e^{(v)}\left(x_{0}, \rho\right)}=e^{(v)}\left(x_{0},-\rho\right)$.

The proof of Lemma 1 is given in [23, Sec. 2.1].

Let $T_{0}:=T \backslash\left\{e_{0}\right\}$ be the compact graph with the edges $e_{1}, \ldots, e_{p}$ and with the vertices $v_{0}, \ldots, v_{p}$. Denote by $L_{0}$ be the boundary value problem for equation (1) on the graph $T_{0}$ with the matching conditions

$$
y_{j}\left(l_{j}\right)=y_{i}\left(l_{i}\right), i, j=\overline{1, p}, \quad \sum_{j=1}^{p} y_{j}^{\prime}\left(l_{j}\right)=0,
$$

and with the boundary conditions (6). Moreover, denote by $L_{k}, k=\overline{1, p}$, the boundary value problem for equation (1) on the graph $T_{0}$ with the matching conditions (8) and with the boundary conditions

$$
V_{k}\left(y_{k}\right)=0, \quad U_{j}\left(y_{j}\right)=0, j=\overline{1, p} \backslash k .
$$

Consider the functions

$$
G_{0}(\lambda)=\prod_{j=1}^{p} \varphi_{j 1}\left(l_{j}, \lambda\right), \quad g_{0}(\lambda)=G_{0}(\lambda) \sum_{j=1}^{p} \frac{\varphi_{j 1}^{\prime}\left(l_{j}, \lambda\right)}{\varphi_{j 1}\left(l_{j}, \lambda\right)} .
$$

Let $G_{k}(\lambda)$ and $g_{k}(\lambda)$ are obtained from $G_{0}(\lambda)$ and $g_{0}(\lambda)$, respectively, by replacing $\varphi_{k 1}^{(\xi)}\left(l_{k}, \lambda\right)$ with $\varphi_{k 2}^{(\xi)}\left(l_{k}, \lambda\right), \xi=0,1$. The functions $G_{k}(\lambda)$ and $g_{k}(\lambda), k=\overline{0, p}$ are entire in $\lambda$ of order $1 / 2$. Zeros of $g_{k}(\lambda), k=\overline{0, p}$ coincide with the eigenvalues of the boundary value problem $L_{k}$. The function $g_{k}(\lambda)$ is called the characteristic function for $L_{k}$.

Denote

$$
\left.\begin{array}{c}
\Delta(\rho)=G_{0}(\lambda) e^{\prime}(0, \rho)-g_{0}(\lambda) e(0, \rho), \\
\Delta_{k}(\rho)=G_{k}(\lambda) e^{\prime}(0, \rho)-g_{k}(\lambda) e(0, \rho) .
\end{array}\right\}
$$

The next assertion follows from (10) and Lemma 1.

Theorem 1. The functions $\Delta(\rho)$ and $\Delta_{k}(\rho), k=\overline{1, p}$, are analytic in $\Omega_{0}$, and continuous in $\Omega$. For real $\rho \neq 0$,

$$
\overline{\Delta(\rho)}=\Delta(-\rho)
$$

Fix $k=\overline{1, p}$. Let $\Psi_{k}=\left\{\psi_{k j}\right\}_{j=0, p}$ be the solution of equation (1) on the graph $T$ satisfying the matching conditions

$$
\psi_{k j}\left(l_{j}, \lambda\right)=\psi_{k 0}(0, \lambda), j=\overline{1, p}, \quad \sum_{j=1}^{p} \psi_{k j}^{\prime}\left(l_{j}, \lambda\right)=\psi_{k 0}^{\prime}(0, \lambda),
$$

and boundary conditions

$$
U_{j}\left(\psi_{k j}\right)=\delta_{k j}, j=\overline{1, p}, \quad \psi_{k 0}\left(x_{0}, \lambda\right)=O\left(\exp \left(i \rho x_{0}\right)\right), x_{0} \rightarrow \infty .
$$


The function $M_{k}(\lambda):=V_{k}\left(\psi_{k k}\right)$ is called the Weyl function with respect to the boundary vertex $v_{k}$, and the vector $M(\lambda)=\left[M_{k}(\lambda)\right]_{k=1, p}$ is called the Weyl vector. The inverse problem is formulated as follows.

Inverse problem 1. Given the Weyl vector $M(\lambda)$, construct the potential $Q$ on the graph $T$ and the vector $h$.

We mention that the notion of the Weyl vector $M$ is a generalization of the notion of the Weyl function (m-function) for the classical Sturm-Liouville operator on an interval, and Inverse problem 1 is a generalization of the classical inverse problems for Sturm-Liouville operator from the Weyl function, and (which is equivalent) from the spectral measure (see [23, Ch.1]). Inverse problem 1 will be solved in Section 4.

Taking (13) into account we infer that the Weyl solution $\Psi_{k}=\left\{\psi_{k j}\right\}_{j=\overline{0, p}}$ has the form

$$
\left.\begin{array}{c}
\psi_{k k}\left(x_{k}, \lambda\right)=\varphi_{k 2}\left(x_{k}, \lambda\right)+M_{k}(\lambda) \varphi_{k 1}\left(x_{k}, \lambda\right), \\
\psi_{k j}\left(x_{j}, \lambda\right)=M_{k j}(\lambda) \varphi_{j 1}\left(x_{j}, \lambda\right), \quad j=\overline{1, p} \backslash k, \\
\psi_{k 0}\left(x_{0}, \lambda\right)=M_{k 0}(\lambda) e\left(x_{0}, \rho\right),
\end{array}\right\}
$$

where $M_{k j}(\lambda), j=\overline{0, p} \backslash k$ do not depend on $x_{j}$. Substituting (14) into (12) we obtain the linear algebraic system $s_{k}$ with respect to $M_{k}(\lambda)$ and $M_{k j}(\lambda), j=\overline{0, p} \backslash k$. The determinant of the system $s_{k}$ is $\Delta(\rho)$. Solving $s_{k}$ by Cramer's rule we get

$$
\begin{gathered}
M_{k}(\lambda)=-\frac{\Delta_{k}(\rho)}{\Delta(\rho)}, \\
M_{k j}(\lambda)=\prod_{s=1}^{p} \varphi_{s 1}\left(l_{s}, \lambda\right) \frac{e(0, \rho)}{\Delta(\rho) \varphi_{j 1}\left(l_{j}, \lambda\right) \varphi_{k 1}\left(l_{k}, \lambda\right)}, \quad j=\overline{1, p} \backslash k, \\
M_{k 0}(\lambda)=\prod_{s=1}^{p} \varphi_{s 1}\left(l_{s}, \lambda\right) \frac{1}{\Delta(\rho) \varphi_{k 1}\left(l_{k}, \lambda\right)} .
\end{gathered}
$$

Denote by $\Lambda:=\left\{\lambda=\rho^{2}: \rho \in \Omega, \Delta(\rho)=0\right\}$ the set of zeros of $\Delta(\rho)$ in $\Omega$. Then $\Lambda=\Lambda^{\prime} \cup \Lambda^{\prime \prime}$, where

$$
\Lambda^{\prime}:=\left\{\lambda=\rho^{2}: \rho \in \Omega_{0}, \Delta(\rho)=0\right\}, \quad \Lambda^{\prime \prime}:=\left\{\lambda=\rho^{2}: \operatorname{Im} \rho=0, \rho \neq 0, \Delta(\rho)=0\right\} .
$$

The following assertion follows from (15), (10) and Theorem 1.

Theorem 2. The Weyl functions $M_{k}(\lambda), k=\overline{1, p}$, are analytic in $\Pi \backslash \Lambda^{\prime}$ and continuous $i n \Pi_{1} \backslash \Lambda$. The set of singularities of $M(\lambda)$ (as an analytic function) coincides with the set $S:=\{\lambda: \lambda \geq$ $0\} \cup \Lambda$. 
Definition 1. The set of singularities of the Weyl vector $M(\lambda)$ is called the spectrum of $L$. The value of the parameter $\lambda$ for which (1) has nontrivial solutions satisfying (5)-(6) and $y_{r}(\infty)=0$ (i.e. $\lim _{x_{r} \rightarrow \infty} y\left(x_{r}\right)=0$ ), are called eigenvalues of $L$, and the corresponding solutions are called eigenfunctions.

Theorem 3. Let $\lambda_{0}=\rho_{0}^{2}, \rho_{0} \in \Omega_{0}$, i.e. $\lambda_{0} \notin[0, \infty)$. For $\lambda_{0}$ to be an eigenvalue of $L$ on $T$, it is necessary and sufficient that $\lambda_{0} \in \Lambda^{\prime}$.

Proof. Let $\lambda_{0}=\rho_{0}^{2} \in \Lambda^{\prime}$. On the graph $T$ we consider the function $Y=\left\{y_{j}\right\}_{j=\overline{0, p}}$ of the form

$$
\left.\begin{array}{c}
y_{j}\left(x_{j}\right)=\alpha_{j 1} \varphi_{j 1}\left(x_{j}, \lambda_{0}\right)+\alpha_{j 2} \varphi_{j 2}\left(x_{j}, \lambda_{0}\right), \quad j=\overline{1, p}, \\
y_{0}\left(x_{0}\right)=\alpha_{0} e\left(x_{0}, \rho_{0}\right) .
\end{array}\right\}
$$

Clearly, $Y$ is a solution of equation (1) for $\lambda=\lambda_{0}$. Substituting (18) into (5) and (6) we obtain a homogeneous linear algebraic system $s_{0}$ with respect to $\alpha_{j 1}, \alpha_{j 2}, j=\overline{1, p}$ and $\alpha_{0}$. The determinant of the system $s_{0}$ is $\Delta\left(\rho_{0}\right)$. Since $\Delta\left(\rho_{0}\right)=0$, it follows that the system $s_{0}$ has a nontrivial solution. This means that $Y=\left\{y_{j}\right\}_{j=\overline{0, p}}$ is an eigenfunction, and $\lambda_{0}$ is an eigenvalue of $L$.

Conversely, let $\lambda_{0}=\rho_{0}^{2} \notin[0, \infty)$ be an eigenvalue of $L$, and let $Y=\left\{y_{j}\right\}_{j=\overline{0, p}}$ be a corresponding eigenfunction. Then $Y$ has the form (18), where $\alpha_{j 1}, \alpha_{j 2}, j=\overline{1, p}$ and $\alpha_{0}$ satisfy the system $s_{0}$. Since $Y$ is not identically zero, it follows that the system $s_{0}$ has a nontrivial solution, and consequently, $\Delta\left(\rho_{0}\right)=0$.

Since $q_{j}\left(x_{j}\right), \omega_{j}$ and $h_{j}$ are real, it is known that the operator $L^{\prime}$ is self-adjoint and bounded from below (see [31]). Together with Theorem 3 this yields that $\Lambda^{\prime} \subset(-\infty, 0)$ lies on the negative real half-axis, and $\Lambda^{\prime}$ is a bounded set of eigenvalues of $L$. Denote by $\Lambda_{0}$ the set of common positive zeros of $g_{0}(\lambda)$ and $G_{0}(\lambda)$.

Theorem 4. $\Lambda^{\prime \prime}=\Lambda_{0}$.

Proof. Let $\lambda_{0} \in \Lambda^{\prime \prime}$. Then $\lambda_{0}=\rho_{0}^{2}>0$ and $\Delta\left(\rho_{0}\right)=0$. It follows from (11) that $\Delta\left(-\rho_{0}\right)=0$. Together with (7) and (10) this yields $g_{0}\left(\lambda_{0}\right)=G_{0}\left(\lambda_{0}\right)=0$, i.e. $\lambda_{0} \in \Lambda_{0}$.

Conversely, let $\lambda_{0} \in \Lambda_{0}$. Then $\lambda_{0}=\rho_{0}^{2}>0$ and $g_{0}\left(\lambda_{0}\right)=G_{0}\left(\lambda_{0}\right)=0$. It follows from (10) that $\Delta\left(\rho_{0}\right)=0$, i.e. $\lambda_{0} \in \Lambda^{\prime \prime}$.

Theorem 5. Let $\lambda_{0}=\rho_{0}^{2}>0$. For $\lambda_{0}$ to be an eigenvalue of $L$, it is necessary and sufficient that $\lambda_{0} \in \Lambda^{\prime \prime}$.

Proof. Let $\lambda_{0}=\rho_{0}^{2}>0$ be an eigenvalue, and let $Y=\left\{y_{j}\right\}_{j=\overline{0, p}}$ be a corresponding eigenfunction. According to (7) the functions $\left\{e\left(x_{0}, \rho_{0}\right), e\left(x_{0},-\rho_{0}\right)\right\}$ form a fundamental system of 
solutions of (1) on $e_{0}$, and consequently, $y_{0}\left(x_{0}\right)=A e\left(x_{0}, \rho_{0}\right)+B e\left(x_{0},-\rho_{0}\right)$. For $x_{0} \rightarrow \infty$ we have $y_{0}\left(x_{0}\right) \sim 0, e\left(x_{0}, \pm \rho_{0}\right) \sim \exp \left( \pm i \rho_{0} x_{0}\right)$. But this is possible only if $A=B=0$, i.e. $y_{0}\left(x_{0}\right) \equiv 0$.

Clearly, $Y^{0}:=Y \backslash\left\{y_{0}\right\}=\left\{y_{j}\right\}_{j=1, p}$ is an eigenfunction of the boundary value problem $L_{0}$ on the graph $T_{0}$, and consequently, $g_{0}\left(\lambda_{0}\right)=0$. Furthermore, one has $U_{j}\left(y_{j}\right)=0$ and $y_{j}\left(l_{j}\right)=0$; hence $y_{j}\left(x_{j}\right)=A_{j} \varphi_{j 1}\left(x_{j}, \lambda_{0}\right)$ and $A_{j} \varphi_{j 1}\left(l_{j}, \lambda_{0}\right)=0, j=\overline{1, p}$. Since $Y^{0}$ is not identically zero, it follows that there exists $m$ such that $A_{m} \neq 0$, and consequently, $\varphi_{m 1}\left(l_{m}, \lambda_{0}\right)=0$. In view of (9), this yields $G_{0}\left(\lambda_{0}\right)=0$, i.e. $\lambda_{0} \in \Lambda_{0}$. Taking Theorem 4 into account we get $\lambda_{0} \in \Lambda^{\prime \prime}$.

Conversely, let $\lambda_{0} \in \Lambda^{\prime \prime}$. According to Theorem 4, $\lambda_{0} \in \Lambda_{0}$, i.e. $g_{0}\left(\lambda_{0}\right)=G_{0}\left(\lambda_{0}\right)=0$. By virtue of (9), there exist $m_{1}, \ldots, m_{s}$ such that $\varphi_{j 1}\left(l_{j}, \lambda_{0}\right)=0$ for $j=m_{1}, \ldots, m_{s}$, and $\varphi_{j 1}\left(l_{j}, \lambda_{0}\right) \neq$ 0 for $j \neq m_{1}, \ldots, m_{s}$. Put $Y=\left\{y_{j}\right\}_{j=\overline{0, p}}$, where $y_{0}\left(x_{0}\right) \equiv 0, y_{j}\left(x_{j}\right) \equiv 0$ for $j \neq m_{1}, \ldots, m_{s}$, and $y_{j}\left(x_{j}\right)=A_{j} \varphi_{j 1}\left(x_{j}, \lambda_{0}\right)$ for $j=m_{1}, \ldots, m_{s}$. Choose the constants $A_{j}$ such that $Y$ satisfies Kirchhoff's condition in $v_{0}$. Then $Y$ is an eigenfunction of $L$, and $\lambda_{0}$ is an eigenvalue of $L$.

Thus, the spectrum of $L$ coincides with $S$, and it consists of the positive half-line $\{\lambda$ : $\lambda \geq 0$, and the discrete real bounded from below set $\Lambda=\Lambda^{\prime} \cup \Lambda^{\prime \prime}$. We note that the set $\Lambda^{\prime \prime}$ of positive eigenvalues can be empty, finite or an infinite unbounded set (see example).

Example. Let $p=2, \omega_{j}=0, l_{j}=1, q_{j}\left(x_{j}\right) \in \mathscr{L}_{2}(0,1)$. Then

$$
\Delta(\rho)=\varphi_{11}(1, \lambda) \varphi_{21}(1, \lambda) e^{\prime}(0, \rho)-\left(\varphi_{11}(1, \lambda) \varphi_{21}^{\prime}(1, \lambda)+\varphi_{11}^{\prime}(1, \lambda) \varphi_{21}(1, \lambda)\right) e(0, \rho),
$$

i.e. $G_{0}(\lambda)=\varphi_{11}(1, \lambda) \varphi_{21}(1, \lambda), g_{0}(\lambda)=\varphi_{11}(1, \lambda) \varphi_{21}^{\prime}(1, \lambda)+\varphi_{11}^{\prime}(1, \lambda) \varphi_{21}(1, \lambda)$. In this case $\Lambda_{0}$ is the set of the common positive eigenvalues of the two scalar problems

$$
-y_{j}^{\prime \prime}+q_{j}\left(x_{j}\right) y_{j}=\lambda y_{j}, x_{j} \in(0,1), y_{j}^{\prime}(0)-h_{j} y_{j}(0)=y_{j}(1)=0, j=1,2 .
$$

It follows from the theory of inverse spectral problems (see, for example, [23, Ch.1]) that for arbitrary sequences of real numbers $\left\{\lambda_{n j}\right\}_{n \geq 1}, j=1,2$, of the form

$$
\lambda_{n j}=\pi^{2}(n+1 / 2)^{2}+c_{j}+\kappa_{n j}, \quad\left\{\kappa_{n j}\right\} \in l_{2}, c_{j} \in \mathbf{R}, j=1,2
$$

there exist real potentials $q_{j} \in \mathscr{L}_{2}(0,1)$ for which $\left\{\lambda_{n j}\right\}_{n \geq 1}, j=1,2$, are the sequences of eigenvalues of the boundary value problems (19). This means that we can choose $q_{1}$ and $q_{2}$ such that the set $\Lambda^{\prime \prime}$ will be either empty, finite or an infinite unbounded set.

\section{Inverse problems}

In this section we study the Inverse problem 1 for Bessel differential operators on the graph $T$. We prove the corresponding uniqueness theorem and provide a constructive procedure for the solution of the inverse problem considered. 
Fix $k=1, \ldots, p$, and consider the following auxiliary inverse problem on the boundary edge $e_{k}$, which is called Problem IP $(k)$.

Problem IP(k). Given $M_{k}(\lambda)$, construct $Q_{k}$ on the edge $e_{k}$ and $h_{k}$.

First we prove the uniqueness theorem for the inverse problem IP(k).

Theorem 6. The specification of the Weyl function $M_{k}$ uniquely determines the potential $Q_{k}$ on the edge $e_{k}$ and the coefficient $h_{k}$.

Proof. On each edge $e_{j}, j=\overline{1, p}$ there exists a special fundamental system of solution $\left\{y_{j k}\left(x_{j}\right.\right.$, $\rho)\}_{k=1,2}$ (see [30]) such that for each fixed $x_{j} \in\left(0, l_{j}\right.$,

$$
y_{j k}^{(\xi)}\left(x_{j}, \rho\right)=\left(\rho R_{k}\right)^{\xi} \exp \left(\rho R_{k} x_{j}\right)[1], R_{1}=i, R_{2}=-i, \xi=0,1, \rho \in \Omega,|\rho| \rightarrow \infty,
$$

and the relations

$$
\begin{aligned}
\varphi_{j m}\left(x_{j}, \lambda\right) & =\sum_{k=1}^{2} D_{j m k}(\rho) y_{j k}\left(x_{j}, \rho\right), \\
y_{j k}\left(x_{j}, \rho\right) & =\sum_{m=1}^{2} B_{j k m}(\rho) \varphi_{j m}\left(x_{j}, \lambda\right)
\end{aligned}
$$

hold, where $[1]=1+O\left(\rho^{-\beta}\right), \beta=\min \left(1,2 v_{1}, \ldots, 2 v_{p}\right),\left[D_{j m k}(\rho)\right]_{m, k=1,2}=\left(\left[B_{j k m}(\rho)\right]_{k, m=1,2}\right)^{-1}$,

$$
\left.\begin{array}{c}
B_{j 1 m}(\rho)=b_{j m} \rho^{\mu_{j m}}[1], B_{j 2 m}(\rho)=b_{j m} \exp \left(i \pi \mu_{j m}\right) \rho^{\mu_{j m}}[1], b_{j 1} b_{j 2}=-\left(i \sin \pi v_{j}\right)^{-1}, \\
D_{j m 1}(\rho)=d_{j m} \exp \left(-i \pi \mu_{j m}\right) \rho^{-\mu_{j m}[1], D_{j m 2}(\rho)=d_{j m} \rho^{-\mu_{j m}}[1],} \\
d_{j 1}=b_{j 2} /(2 i), \quad d_{j 2}=-b_{j 2} /(2 i) .
\end{array}\right\}
$$

Denote $\Omega_{\delta}:=\{\rho: \arg \rho \in[\delta, \pi-\delta]\}, \delta>0$. It follows from (20), (21) and (23) that for each fixed $x_{j} \in\left(0, l_{j}\right], \xi=0,1$,

$$
\left.\begin{array}{c}
\varphi_{j 1}^{(\xi)}\left(x_{j}, \lambda\right)=(2 i)^{-1} b_{j 2} \rho^{v_{j}-1 / 2}(-i \rho)^{\xi} \exp \left(-i \rho x_{j}\right)[1], \quad \rho \in \Omega_{\delta},|\rho| \rightarrow \infty, \\
\varphi_{j 2}^{(\xi)}\left(x_{j}, \lambda\right)=-(2 i)^{-1} b_{j 1} \rho^{-v_{j}-1 / 2}(-i \rho)^{\xi} \exp \left(-i \rho x_{j}\right)[1], \quad \rho \in \Omega_{\delta},|\rho| \rightarrow \infty .
\end{array}\right\}
$$

Substituting (24) into (10) and using Lemma 1, we get for $\rho \in \Omega_{\delta},|\rho| \rightarrow \infty$ :

$$
\left.\begin{array}{c}
\Delta(\rho)=\frac{(p+1)(i \rho)}{(2 i)^{p}}\left(\prod_{j=1}^{p} b_{j 2}\right) \rho^{v_{1}+\ldots+v_{p}-p / 2} \exp \left(-i \rho\left(l_{1}+\ldots+l_{p}\right)\right)[1], \\
\Delta_{k}(\rho)=-\frac{(p+1)(i \rho) b_{k 1}}{(2 i)^{p} b_{k 2}}\left(\prod_{j=1}^{p} b_{j 2}\right) \rho^{v_{1}+\ldots+v_{p}-p / 2-2 v_{k}} \exp \left(-i \rho\left(l_{1}+\ldots+l_{p}\right)\right)[1] .
\end{array}\right\}
$$


By virtue of (15)-(17) and (24)-(25) one has for $k=\overline{1, p}, \rho \in \Omega_{\delta},|\rho| \rightarrow \infty$ :

$$
\left.\begin{array}{c}
M_{k}(\lambda)=\frac{b_{k 1}[1]}{b_{k 2} \rho^{2 v_{k}}}, \\
M_{k j}(\lambda)=\frac{(2 i)^{2} \exp \left(i \rho\left(l_{k}+l_{j}\right)\right)[1]}{i(p+1) b_{k 2} b_{j 2} \rho^{v_{k}+v_{j}}}, j \neq k .
\end{array}\right\}
$$

Using the fundamental system of solutions $\left\{y_{j k}\left(x_{j}, \rho\right)\right\}_{k=1,2}$ we have

$$
\left.\begin{array}{c}
\psi_{k j}\left(x_{j}, \lambda\right)=a_{k j 1}(\rho) y_{j 1}\left(x_{j}, \rho\right)+a_{k j 2}(\rho) y_{j 2}\left(x_{j}, \rho\right), j=\overline{1, p}, \\
\psi_{k 0}\left(x_{0}, \lambda\right)=a_{k 0}(\rho) e\left(x_{0}, \rho\right) .
\end{array}\right\}
$$

It follows from (22) and (27) that

$$
\psi_{k j}\left(x_{j}, \lambda\right)=\sum_{s=1}^{2} a_{k j s}(\rho) \sum_{m=1}^{2} B_{j s m}(\rho) \varphi_{j m}\left(x_{j}, \lambda\right)=\sum_{m=1}^{2} \varphi_{j m}\left(x_{j}, \lambda\right) \sum_{s=1}^{2} a_{k j s}(\rho) B_{j s m}(\rho) .
$$

Comparing with (14) we obtain

$$
\left.\begin{array}{c}
a_{k j 1}(\rho) B_{j 11}(\rho)+a_{k j 2}(\rho) B_{j 21}(\rho)=M_{k j}(\lambda), \\
a_{k j 1}(\rho) B_{j 12}(\rho)+a_{k j 2}(\rho) B_{j 22}(\rho)=\delta_{k j},
\end{array}\right\}
$$

where $M_{k k}(\lambda):=M_{k}(\lambda)$. Solving this linear algebraic system and using (23), (26), we get for $\rho \in \Omega_{\delta},|\rho| \rightarrow \infty:$

$$
\left.\begin{array}{c}
a_{k k 1}(\rho)=\frac{[1]}{b_{k 2} \rho^{v_{k}+1 / 2}}, \\
O\left(\rho^{-v_{k}-1 / 2} \exp \left(i \rho\left(l_{k}+l_{j}\right)\right)\right), j \neq k .
\end{array}\right\}
$$

We note that system (28) is not convenient for calculating $a_{k j 2}(\rho)$. In order to estimate $a_{k j 2}(\rho)$ we substitute (27) into the matching conditions (12):

$$
\left.\begin{array}{c}
a_{k j 1}(\rho) y_{j 1}\left(l_{j}, \rho\right)+a_{k j 2}(\rho) y_{j 2}\left(l_{j}, \rho\right)-a_{k 0}(\rho) e(0, \rho)=0, j=\overline{1, p} \\
\sum_{j=1}^{p}\left(a_{k j 1}(\rho) y_{j 1}^{\prime}\left(l_{j}, \rho\right)+a_{k j 2}(\rho) y_{j 2}^{\prime}\left(l_{j}, \rho\right)\right)-a_{k 0}(\rho) e^{\prime}(0, \rho)=0 .
\end{array}\right\}
$$

Taking (30), (29) and (20) into account we infer

$$
a_{k k 2}(\rho)=O\left(\rho^{-v_{k}-1 / 2} \exp \left(2 i \rho l_{k}\right)\right), \quad \rho \in \Omega_{\delta},|\rho| \rightarrow \infty .
$$

Together with (27) and (29) this yields, in particular,

$$
\psi_{k k}^{(\xi)}\left(x_{k}, \lambda\right)=\frac{(i \rho)^{\xi} \exp \left(i \rho x_{k}\right)[1]}{b_{k 2} \rho^{v_{k}+1 / 2}}, x_{k} \in\left(0, l_{k}\right), \xi=0,1, \rho \in \Omega_{\delta},|\rho| \rightarrow \infty .
$$


Together with $L=L(Q, h)$ we consider a boundary value problem $\tilde{L}=L(\tilde{Q}, \tilde{h})$ of the same form but with $\tilde{Q}$ and $\tilde{h}$ instead of $Q$ and $h$. Everywhere below if a symbol $\alpha$ denotes an object related to $L$, then $\tilde{\alpha}$ will denote the analogous object related to $\tilde{L}$.

According to assumptions of the theorem we assume that $M_{k}(\lambda) \equiv \tilde{M}_{k}(\lambda)$. Consider the functions

$$
\left.\begin{array}{l}
P_{k 1}\left(x_{k}, \lambda\right)=\varphi_{k 1}\left(x_{k}, \lambda\right) \tilde{\psi}_{k k}^{\prime}\left(x_{k}, \lambda\right)-\psi_{k k}\left(x_{k}, \lambda\right) \tilde{\varphi}_{k 1}^{\prime}\left(x_{k}, \lambda\right), \\
P_{k 2}\left(x_{k}, \lambda\right)=\psi_{k k}\left(x_{k}, \lambda\right) \tilde{\varphi}_{k 1}\left(x_{k}, \lambda\right)-\varphi_{k 1}\left(x_{k}, \lambda\right) \tilde{\psi}_{k k}\left(x_{k}, \lambda\right) .
\end{array}\right\}
$$

Using (24), (31) and (32) we infer

$$
P_{k s}\left(x_{k}, \lambda\right)=\delta_{1 s}+O\left(\rho^{-\beta}\right), \quad x_{k} \in\left(0, l_{k}\right), \rho \in \Omega_{\delta},|\rho| \rightarrow \infty .
$$

Since $\left\langle\varphi_{k 1}\left(x_{k}, \lambda\right), \varphi_{k 2}\left(x_{k}, \lambda\right)\right\rangle \equiv 1$, it follows from (14) that

$$
\left\langle\varphi_{k 1}\left(x_{k}, \lambda\right), \psi_{k k}\left(x_{k}, \lambda\right)\right\rangle \equiv 1
$$

By virtue of (32) this yields

$$
P_{k 1}\left(x_{k}, \lambda\right) \tilde{\varphi}_{k 1}\left(x_{k}, \lambda\right)+P_{k 2}\left(x_{k}, \lambda\right) \tilde{\varphi}_{k 1}^{\prime}\left(x_{k}, \lambda\right)=\varphi_{k 1}\left(x_{k}, \lambda\right) .
$$

Substituting (14) into (32) we obtain

$$
\begin{gathered}
P_{k 1}\left(x_{k}, \lambda\right)=\varphi_{k 1}\left(x_{k}, \lambda\right) \tilde{\varphi}_{k 2}^{\prime}\left(x_{k}, \lambda\right)-\varphi_{k 2}\left(x_{k}, \lambda\right) \tilde{\varphi}_{k 1}^{\prime}\left(x_{k}, \lambda\right) \\
+\left(\tilde{M}_{k}(\lambda)-M_{k}(\lambda)\right) \varphi_{k 1}\left(x_{k}, \lambda\right) \tilde{\varphi}_{k 1}^{\prime}\left(x_{k}, \lambda\right), \\
P_{k 2}\left(x_{k}, \lambda\right)=\varphi_{k 2}\left(x_{k}, \lambda\right) \tilde{\varphi}_{k 1}\left(x_{k}, \lambda\right)-\varphi_{k 1}\left(x_{k}, \lambda\right) \tilde{\varphi}_{k 2}\left(x_{k}, \lambda\right) \\
+\left(M_{k}(\lambda)-\tilde{M}_{k}(\lambda)\right) \varphi_{k 1}\left(x_{k}, \lambda\right) \tilde{\varphi}_{k 1}\left(x_{k}, \lambda\right) .
\end{gathered}
$$

Since $M_{k}(\lambda) \equiv \tilde{M}_{k}(\lambda)$, it follows that for each fixed $x_{k}$, the functions $P_{k s}\left(x_{k}, \lambda\right)$ are entire in $\lambda$ of order $1 / 2$. Together with (33) this yields $P_{k 1}\left(x_{k}, \lambda\right) \equiv 1, P_{k 2}\left(x_{k}, \lambda\right) \equiv 0$. Substituting these relations into (34) we get $\varphi_{k 1}\left(x_{k}, \lambda\right) \equiv \tilde{\varphi}_{k 1}\left(x_{k}, \lambda\right)$ for all $x_{k}$ and $\lambda$, and consequently, $Q_{k}\left(x_{k}\right)=$ $\tilde{Q}_{k}\left(x_{k}\right)$ a.e. on $\left(0, l_{k}\right)$ and $h_{k}=\tilde{h}_{k}$.

Using the method of spectral mappings [25] for the Sturm-Liouville operator on the edge $e_{k}$ one can get a constructive procedure for the solution of the inverse problem $I P(k)$. Here we only explain ideas briefly; for details and proofs see [25]. Choose $\tilde{L}$ such that $\tilde{v}_{k}=v_{k}$. Denote by $\lambda^{\prime}$ the minimal eigenvalue of $L$ and $\tilde{L}$, and take a fixed $\delta>0$. In the $\lambda$-plane we consider the contour $\theta$ (with counterclockwise circuit) of the form $\theta=\theta^{+} \cup \theta^{-} \cup \theta^{\prime}$, where $\theta^{ \pm}=\{\lambda$ : $\left.\pm \operatorname{Im} \lambda=\delta ; \operatorname{Re} \lambda \geq \lambda^{\prime}\right\}, \theta^{\prime}=\left\{\lambda: \lambda-\lambda^{\prime}=\delta \exp (i \alpha), \alpha \in(\pi / 2,3 \pi / 2)\right\}$. For each fixed $x_{k} \in\left(0, l_{k}\right)$, the function $\varphi_{k 1}\left(x_{k}, \lambda\right)$ is the unique solution of the following linear integral equation

$$
\varphi_{k 1}\left(x_{k}, \lambda\right)=\tilde{\varphi}_{k 1}\left(x_{k}, \lambda\right)+\frac{1}{2 \pi i} \int_{\theta} \tilde{D}_{k}\left(x_{k}, \lambda, \mu\right) \varphi_{k 1}\left(x_{k}, \mu\right) d \mu
$$


where

$$
\tilde{D}_{k}(x, \lambda, \mu)=\frac{\left\langle\tilde{\varphi}_{k 1}\left(x_{k}, \lambda\right), \tilde{\varphi}_{k 1}\left(x_{k}, \mu\right)\right\rangle}{\lambda-\mu} \hat{M}_{k}(\mu) d t, \quad \hat{M}_{k}(\mu):=M_{k}(\mu)-\tilde{M}_{k}(\mu) .
$$

The potential $Q_{k}$ on the edge $e_{k}$ and the coefficient $h_{k}$ can be constructed from the solution of the integral equation (35):

$$
Q_{k}\left(x_{k}\right)=\lambda+\frac{\varphi_{k 1}^{\prime \prime}\left(x_{k}, \lambda\right)}{\varphi_{k 1}\left(x_{k}, \lambda\right)}, \quad h_{k}=\sigma_{k 2}\left(\varphi_{k 1}\right)
$$

The solution of Inverse problem 1 can be found by the following algorithm.

\section{Algorithm 1.}

(1) For each fixed $k=\overline{1, p}$, we solve $I P(k)$ and find the potential $Q_{k}$ on the edge $e_{k}$ and the coefficient $h_{k}$.

(2) Calculate $\varphi_{k m}\left(x_{k}, \lambda\right), m=1,2$, and $\psi_{k k}\left(x_{k}, \lambda\right)$ via (14).

(3) Find $\psi_{k j}\left(l_{k}, \lambda\right)$ for $j, k=\overline{1, p}$ using (12).

(4) Construct $M_{k j}(\lambda), j=\overline{1, p} \backslash k$ by (14).

(5) Calculate $\Delta(\rho) / e(0, \rho)$ from (16).

(6) Construct $G_{0}(\lambda)$ and $g_{0}(\lambda)$ via (9).

(7) Find $M_{0}(\lambda):=e^{\prime}(0, \rho) / e(0, \rho)$ using (10).

(8) Construct the potential $Q$ on $e_{0}$ by solving classical inverse Sturm-Liouville problem on the half-line from the Weyl function $M_{0}(\lambda)$ (see [23]).

Thus, executing Algorithm 1 we obtain the solution of Inverse problem 1 and prove its uniqueness.

\section{Acknowledgement}

This research was supported in part by Grants 10-01-00099 and 10-01-92001-NSC of Russian Foundation for Basic Research and Taiwan National Science Council.

\section{References}

[1] M. D. Faddeev and B. S. Pavlov, Model of free electrons and the scattering problem, Teor. Math. Fiz., 55, (1983), 257-269 (Russian); English transl. in Theor. Math. Phys., 55 (1983), 485-492.

[2] J. E. Langese, G. Leugering and J. P. G. Schmidt, Modelling, Analysis and Control of Dynamic Elastic MultiLink Structures, Birkhäuser, Boston, 1994.

[3] T. Kottos and U. Smilansky, Quantum chaos on graphs, Phys. Rev. Lett., 79 (1997), 4794-4797.

[4] A. Sobolev and M. Solomyak, Schrödinger operator on homogeneous metric trees: spectrum in gaps, Rev. Math. Phys., 14, (2002), 421-467. 
[5] Yu. V. Pokornyi and A. V. Borovskikh, Differential equations on networks (geometric graphs), J. Math. Sci. (N.Y.), 119, (2004), 691-718.

[6] Yu. Pokornyi and V. Pryadiev, The qualitative Sturm-Liouville theory on spatial networks, J. Math. Sci. (N.Y.), 119 (2004), 788-835.

[7] M. I. Belishev, Boundary spectral inverse problem on a class of graphs (trees) by the BC method, Inverse Problems 20, (2004), 647-672.

[8] V. A. Yurko, Inverse spectral problems for Sturm-Liouville operators on graphs, Inverse Problems 21 (2005), 1075-1086.

[9] B. M. Brown and R. Weikard, A Borg-Levinson theorem for trees, Proc. R. Soc. Lond. Ser. A Math. Phys. Eng. Sci., 461, (2005), 3231-3243.

[10] P. Kurasov and M. Nowaczyk, Inverse spectral problem for quantum graphs, J. Phys. A, 38 (2005), 4901-4915.

[11] V. Pivovarchik, Inverse problem for the Sturm-Liouville equation on a simple graph, SIAM J. Math. Anal., 32, (2000), 801-819.

[12] V. A. Yurko, Inverse problems for Sturm-Liouville operators on bush-type graphs, Inverse Problems 25, (2009), 105008, 14pp.

[13] V. A. Yurko, Uniqueness of recovering Sturm-Liouville operators on A-graphs from spectra, Results in Mathematics, 55, (2009), 199-207.

[14] V. A. Yurko, An inverse problem for Sturm-Liouville operators on arbitrary compact spatial networks, Doklady Akad. Nauk, 432, (2010), 318-321; English transl: Doklady Mathematics 81, (2010), 410-413.

[15] N. I. Gerasimenko, Inverse scattering problem on a noncompact graph, Teoret. Mat. Fiz., 74 (1988), 187-200 (Russian); English transl. in Theor. Math. Phys., 75 (1988), 460-470.

[16] G. Freiling and V. A. Yurko, Inverse spectral problems for Sturm-Liouville operators on noncompact trees, Results in Math., 50 (2007), 195-212.

[17] V. Pivovarchik and Y. Latushkin, Scattering in a forked-shaped waveguide, Integral Equat. Oper. Theory 61 (2008), 365-399.

[18] V. A. Marchenko, Sturm-Liouville operators and their applications, "Naukova Dumka", Kiev, 1977; English transl., Birkhäuser, 1986.

[19] B. M. Levitan, Inverse Sturm-Liouville problems, Nauka, Moscow, 1984; English transl., VNU Sci.Press, Utrecht, 1987.

[20] R. Beals, P. Deift and C. Tomei, Direct and Inverse Scattering on the Line, Math. Surveys and Monographs, v.28. Amer. Math. Soc. Providence: RI, 1988.

[21] J. Pöschel and E. Trubowitz, Inverse Spectral Theory, New York, Academic Press, 1987.

[22] J. R. McLaughlin, Analytical methods for recovering coefficients in differential equations from spectral data, SIAM Rev., 28 (1986), 53-72.

[23] G. Freiling and V. A. Yurko, Inverse Sturm-Liouville Problems and their Applications, NOVA Science Publishers, New York, 2001.

[24] K. Chadan, D. Colton, L. Paivarinta and W. Rundell, An introduction to inverse scattering and inverse spectral problems, SIAM Monographs on Mathematical Modelling and Computation, SIAM, Philadelphia, PA, 1997.

[25] V. A. Yurko, Method of Spectral Mappings in the Inverse Problem Theory, Inverse and Ill-posed Problems Series, VSP, Utrecht, 2002.

[26] V. A. Yurko, Inverse Spectral Problems for Differential Operators and their Applications, Gordon and Breach, Amsterdam, 2000, 253pp.

[27] A. G. Ramm, Inverse problems, Mathematical and Analytical Techniques with Applications to Engineering, Springer, New York, 2005.

[28] B. M. Levitan and I. S. Sargsyan, Introduction to Spectral Theory, AMS Transl. of Math. Monogr. 39, Providence, 1975.

[29] G. Freiling and V. A. Yurko, Inverse problems for differential operators with singular boundary conditions, Mathematishe Nachrichten 278, (2005), 1561-1578.

[30] V. A. Yurko, Inverse problem for differential equations with a singularity, Differ. Uravneniya, 28, (1992), 13551362 (Russian); English transl. in Diff. Equations, 28 (1992), 1100-1107. 
[31] N. A. Naimark, Linear Differential Operators, 2nd ed., Nauka, Moscow, 1969; English transl. of 1st ed., Parts I,II, Ungar, New York, 1967, 1968.

Department of Mathematics, Saratov State University, Astrakhanskaya 83, Saratov 410012, Russia.

E-mail: yurkova@info.sgu.ru 